\title{
Clinical characteristics and fetal outcomes in women with epilepsy with planned and unplanned pregnancy: a retrospective study
}

\author{
Yaoyao Zhang ${ }^{1}$, Changgeng Song ${ }^{1}$, Xuan Wang ${ }^{1}$, Yongli Jiang ${ }^{1}$, Jingjing Zhao ${ }^{1}$, Fang \\ Yuan $^{1}$, Xiai Yang ${ }^{1}$, Fang Yang ${ }^{1}$, and Wen Jiang ${ }^{2}$ \\ ${ }^{1}$ Department of Neurology, Xijing Hospital, Fourth Military Medical University \\ ${ }^{2}$ Affiliation not available
}

May 5, 2020

\begin{abstract}
Objective: To compare the antiepileptic drug (AED) patterns, seizure control, and folic acid supplementation between planned and unplanned pregnancy in women with epilepsy (WWE), and investigate the effects of planned pregnancy on fetal outcomes. Design: Retrospective study. Setting: A prospectively collected database from Feb 2010 to Dec 2018 in Xijing Hospital. Populations: Pregnant WWE. Main Outcome Measures: Clinical characteristics and fetal outcomes. Results: 477 pregnancies were enrolled: 188 planned pregnancies (39.4\%) and 289 unplanned pregnancies (60.6\%). The education level of the unplanned group was lower than that of the planned group $(\mathrm{P}<0.001)$. Among the planned group, $66.0 \%$ took AED monotherapy, and $32.4 \%$ received polytherapy. In the unplanned group, $58.1 \%$ did not take AEDs, $28.0 \%$ took monotherapy, and $12.8 \%$ received polytherapy. Compared with the unplanned group, The planned group had less generalized tonic-clonic seizures (2 [1-4] vs. 1 $[1-2] ; \mathrm{P}=0.002)$ and a higher proportion of being seizure-free $(41.0 \%$ vs. $22.8 \% ; \mathrm{P}<0.001)$. All planned pregnancies took folic acid while $39.8 \%$ of unplanned pregnancies never took it $(\mathrm{P}<0.001)$. The planned group showed significantly less adverse fetal outcomes than the unplanned group: induced abortions $(2.7 \%$ vs. $13.5 \%$; P $<0.001)$, preterm births $(3.3 \%$ vs. $20.4 \%$; $\mathrm{P}<0.001)$, and major congenital malformations within one year of delivery $(1.6 \%$ vs. $7.5 \% ; \mathrm{P}=0.016)$. Regression analysis demonstrated that pregnancy planning was independently associated with adverse fetal outcomes (adjusted odds ratio, 0.14; 95\% confidence interval, 0.08-0.27; P < 0.001). Conclusions: Planned-pregnancy could benefit both WWE and their offspring.
\end{abstract}

\section{Introduction}

Epilepsy is a common neurological disease that affects approximately 12.5 million women of childbearing age worldwide. ${ }^{1}$ Compared with healthy individuals, women with epilepsy (WWE) often have increased risk of pregnancy complications, for example, gestational hypertension, preeclampsia, post-partum hemorrhage, antepartum hemorrhage, placental abruption premature rupture of membranes, preterm labor, and cesarean section. ${ }^{2-6}$ Furthermore, infants of WWE who take antiepileptic drugs (AEDs) are at additional risk of major congenital malformations (MCMs), ${ }^{7,8}$ neurodevelopmental delays, ${ }^{9}$ and low birth weights. ${ }^{10}$ Therefore, preconception and prenatal care to ensure a healthy pregnancy, labor, and delivery for WWE is a critical challenge.

Studies have demonstrated that good seizure control, optimized AEDs types and doses, and appropriate folic acid supplementation can benefit both WWE and their children. ${ }^{11-14}$ Notably, the newer generation AEDs such as lamotrigine (LTG), levetiracetam (LEV), and oxcarbazepine (OXC) in monotherapy have been reported to have a low risk of congenital malformations, comparable to the range as reported in unexposed offspring. ${ }^{15,16}$ By contrast, some AEDs, for example, valproate (VPA), have substantially high risks of 
teratogenesis. ${ }^{17}$ Thus, preconception counseling and pregnancy management, namely, pregnancy planning, may be effective strategies to decrease the risk of pregnancy complications and offspring malformations.

Currently, a large proportion of pregnancies in WWE are unplanned. Data from the Epilepsy Birth Control Registry (EBCR) indicated that the percentage of unplanned pregnancies in WWE was up to $65 \%,{ }^{18}$ and that unplanned pregnancy in WWE may double the risk for spontaneous fetal loss (SFL). ${ }^{19}$ However, the association between planned and unplanned pregnancy and offspring's MCMs has not been studied. Here, based on the prospectively registered database for WWE and pregnancy in Xijing Hospital, we analyzed the differences in AED treatment patterns, seizure control, and folic acid supplementation between planned and unplanned pregnancy and investigated the effect of planned pregnancy in WWE on fetal outcomes.

\section{Methods}

\section{Study design and setting}

This study was a retrospective study based on a prospectively collected database including WWE with pregnancy (age: 18-45 years) in the department of neurology in Xijing Hospital, Shaanxi Province, China, a tertiary academic hospital. This study was approved by the Ethics Committee of Xijing Hospital (KY20163380 - 1) and was in compliance with the Helsinki Declaration.

\section{Patients}

From Feb 2010 to Dec 2018, consecutive cases of WWE with pregnancy registered at Xijing Hospital were retrospectively reviewed. Patients who fulfilled the following criteria were excluded from the study: a) WWE developing epilepsy during or after pregnancy, b) WWE who were not followed up as long as one year after delivery at the last follow-up (May 2019), c) WWE who were lost in follow-up, d) patients whose data were incomplete, and e) WWE exposed to other teratogenic substances. All the enrolled WWE were divided into two groups: planned-pregnancy group and unplanned-pregnancy group. Planned pregnancy was defined as preconception counseling with epileptologists in a face-to-face manner to optimize the type and dose of AEDs and folic acid supplementation and regularly followed up according to changes in clinical status. The unplanned-pregnancy group contained all the pregnancies not included in the planned-pregnancy group, such as the WWE with unexpected pregnancies, who thus had no preconception counseling, the WWE who consulted epileptologists after conception, or the WWE who could not be regularly followed up during pregnancy.

\section{Data collection and analysis}

Clinical data were extracted from the detailed documentation in the database, including age of seizure onset, age at conception, residence, education level, types of epilepsy before pregnancy, AED treatment patterns in the first trimester, generalized tonic-clonic seizure (GTCS) frequency during pregnancy, folic acid supplementation, and fetal outcomes (induced abortion, SFL, live birth, preterm birth, cesarean section, and MCM within one year of delivery). Clinical data were reviewed and compared between the planned and unplanned pregnancy groups by experienced epileptologists. The classification of epilepsy was in accordance with the new 2017 International League Against Epilepsy guideline. ${ }^{20}$ Appropriate folic acid supplementation was defined as the use of folic acid from four weeks before to 12 weeks after conception with a minimum daily dosage of $0.4 \mathrm{mg} .{ }^{12,} 21$

\section{Statistical analysis}

Continuous variables were expressed as mean and standard deviation for normally distributed data and median and inter-quartile range for skewed data. Categorical variables were expressed as counts and percentages. Chi-square tests with Bonferroni post hoc were used to compare proportions among categorical variables. Mann-Whitney U tests were used to analyze the non-normal distribution variables. Binary logistic regression was conducted to analyze the association with adverse fetal outcomes (combining induced abortions, SFLs, preterm births, and MCMs) using pregnancy planning, age at conception, appropriate folic acid supplementation, whether taking VPA during pregnancy, and GTCS frequency during pregnancy as 
covariates. Two-tailed $P$ values $<0.05$ were considered statistically significant. In the post hoc of the chi-square test, $P$ boundary value $\left(\alpha^{\prime}\right)$ was adjusted according to the Bonferroni method. Statistical analysis was performed using SPSS 24.0 (IBM Inc.).

\section{Results}

558 pregnancies in $467 \mathrm{WWE}$ were retrospectively reviewed in this study. Because 81 pregnancies fulfilled the exclusion criteria of the study, 477 pregnancies in $401 \mathrm{WWE}$ were gathered for final analysis. There were 188 pregnancies $(188 / 477,39.4 \%)$ in the planned-pregnancy group and 289 pregnancies $(289 / 477,60.6 \%)$ in the unplanned-pregnancy group. The flowchart of the study is presented in Figure 1.

\section{Baseline characteristics of WWE}

Baseline characteristics of WWE in the planned and unplanned pregnancy group are summarized in Table 1. WWE in the planned group had a younger age of seizure onset (16 [12-20]) than the unplanned-pregnancy group (18 [13-22]; $P=0.016)$. There were no significant differences in the age at conception $(P=0.173$, Mann-Whitney U test) and types of epilepsy $(P=0.479$, Chi-square test) between the two groups. The unplanned-pregnancy group tended to have a higher proportion living in rural areas compared with that of the planned group (64.4\% vs $56.4 \% ; P=0.081$, Chi-square test). As expected, of the two groups, the unplanned-pregnancy group had a lower education level $(P=0.003$, Chi-square test $)$, with $1.7 \%$ illiteracy and $17.6 \%$ primary school education; in the planned group, no one was illiterate, and $91.5 \%$ of the cases of pregnancy received a middle school education or above. Folic acid supplementation patterns were significantly different $(P<0.001$, Chi-square test) between the planned and unplanned pregnancy groups: $39.8 \%$ in the unplanned group did not take folic acid supplementation during pregnancy, and $24.2 \%$ received inappropriate folic acid supplementation. By contrast, $100 \%$ in the planned group took folic acid supplementation, and $81.9 \%$ received appropriate folic acid supplementation.

\section{AEDs patterns and seizure activity during pregnancy}

In Table 1, the AED treatment patterns in the first trimester were significantly different between the planned and unplanned pregnancy groups $(P<0.001$, Chi-square test). In the planned-pregnancy group, $66.0 \%$ of the pregnancies in WWE remained in monotherapy in the first trimester, $32.4 \%$ in polytherapy, and $1.6 \%$ with no AEDs. In the unplanned-pregnancy group, 58.1\% did not take AEDs, $28.0 \%$ remained in monotherapy, $12.8 \%$ remained in polytherapy, and $1.0 \%$ were treated with traditional Chinese medicine. No pregnancy in the planned group was prescribed VPA monotherapy during the first trimester, but in the unplanned group, $4.8 \%$ received VPA monotherapy ( $P=0.002$, Chi-square test). The proportion of WWE taking VPA in polytherapy was similar between the planned and unplanned groups $(5.9 \%$ vs $5.5 \% ; P=0.884$, Chisquare test). The most commonly prescribed AEDs as monotherapy was LTG (52.4\%), LEV (24.2\%), and OXC $(18.5 \%)$ in the planned group, and LTG (30.9\%), VPA (17.3\%), and carbamazepine (CBZ, 14.8\%) in the unplanned group respectively (Figure 2). For polytherapy, the most commonly prescribed combination pattern in the planned group was LEV plus OXC (21.3\%), followed by LTG plus LEV (18.0\%) and LTG plus CBZ (18.0\%). Whereas in the unplanned group, the most common pattern was LTG plus VPA (18.9\%), followed by CBZ plus phenobarbital (PB) (10.8\%) and CBZ plus VPA (10.8\%).

During pregnancy, the number of GTCS in the unplanned group (2 [1-4]) was significantly more than that in the planned group (1 [1-2]; $P=0.002$, Mann-Whitney U test). In addition, up to $41.0 \%$ of pregnancies in the planned group achieved being seizure free during pregnancy, which was significantly more than that in the unplanned group $(22.8 \% ; P<0.001$, Chi-square test $)$.

\section{Fetal outcomes}

Fetal outcomes are presented in Table 2. More pregnancies had a preterm birth in the unplanned-pregnancy group than in the planned-pregnancy group (20.4\% vs. $3.3 \% ; P<0.001$, Chi-square test). The cesarean section rate was higher in the unplanned group than in the planned group $(56.3 \%$ vs. $45.1 \% ; P=0.023$, Chi-square test). Compared with the unplanned-pregnancy group, the planned-pregnancy group had a significantly higher live birth rate $(96.8 \%$ vs. $83.0 \% ; P<0.001$; Chi-square test), and a lower induced 
abortion rate $(2.7 \%$ vs. $13.5 \% ; P<0.001$; Chi-square test). The planned-pregnancy group seemed to have less SFL than the unplanned-pregnancy group, though statistical significance was not reached $(0.5 \%$ vs. $3.5 \% ; P=0.077$, Chi-square test).

The MCM rate within one year after delivery among live births in the unplanned group was significantly higher than that in the planned-pregnancy group ( $7.5 \%$ vs. $1.6 \% ; P=0.006$, Chi-square test). All the three MCM cases in the planned-pregnancy group were congenital cardiac defects, of which one was ventricular septal defect, one was patent foramen ovale, and the other one was atrial septal defect. Of the $18 \mathrm{MCMs}$ in the unplanned-pregnancy group, there were nine cases with congenital cardiac defects, two cases with bifid spine, two cases with cleft lip and cleft palate, two cases with hypospadias, one case with conjoined twins, one case with club foot, and one case with anotia. Detailed AED treatment patterns and folic acid supplementation for the 21 pregnancies with MCMs in the planned and unplanned group are presented in Table S1 in the supplement. All cases of MCMs had no family history of malformation.

Next, we pooled the pregnancies with either induced abortion, SFL, preterm birth, or offspring with MCMs together and referred to them as pregnancies with adverse fetal outcomes. Regression analysis demonstrated that the risk of adverse fetal outcomes was significantly lower for the planned pregnancies when planning was entered alone (OR, $0.13 ; 95 \% \mathrm{CI}, 0.07-0.23 ; P<0.001)$ or adjusted for age at conception, whether taking VPA in either monotherapy or polytherapy in the first trimester, the frequency of GTCS during pregnancy, and whether receiving appropriate folic acid supplementation (OR, 0.14; 95\% CI, 0.08-0.27; $P<0.001$ ). Regression analysis also identified whether taking VPA during pregnancy (OR, 4.34; 95\% CI, 2.09-9.00; $P$ $<0.001$ ), frequency of GTCS during pregnancy (OR, 1.16; 95\% CI, 1.03-1.3; $P=0.012$ ) but not age at conception (OR, 1.00; 95\% CI, 0.94-1.07; $P=0.935)$, and appropriate folic acid supplementation (OR, 1.02; $95 \% \mathrm{CI}, 0.63-1.66 ; P=0.941)$ were significant independent associated factors.

\section{Discussions}

In this study, we observed that approximately $60 \%$ of pregnancies in WWE were unplanned, which was more common among WWE with a lower education level. More importantly, we found that planned pregnancy was associated with more optimized AEDs treatment patterns, better seizure control, more appropriate folic acid supplementation and less adverse fetal outcomes with pregnancy planning as independent risk factor. Our results indicate that pregnancy planning may be a critical strategy for WWE to fulfill satisfactory seizure control and improve their chances of having a healthy baby.

Although pregnancy planning is critical for WWE, more than half of the pregnancies in WWE were unplanned. The result from EBCR web-based survey of 1,144 WWE showed that $65.0 \%$ of their pregnancies were unintended. ${ }^{18}$ The data from the Pregnancy Risk Assessment Monitoring System (PRAMS), including 13 states in the United States from 2009 to 2014, demonstrated that 55\% of WWE pregnancies were unintended. ${ }^{22}$ They reported that unintended pregnancies were more common in WWE with younger ages, lower incomes, or who were members of racial minority groups or Hispanic. ${ }^{22}$ The population in our study was Han Chinese. We found that WWE in the unplanned-pregnancy group had a lower level of education and tended to reside in areas that were more rural compared with the planned-pregnancy group. These patients often have limited access to medical advice.

The greatest concern for WWE is the teratogenicity of AEDs. Accumulating evidence suggests that different AEDs and dosages have different teratogenic risks, ${ }^{23-25}$ and that the newer generation AEDs, such as LTG, LEV, and OXC, are not associated with significantly increased risks of congenital malformations compared with no AED exposure control. ${ }^{15,16}$ In this study, we found that more than $50 \%$ of pregnancies in the unplanned-pregnancy group were not prescribed AEDs during pregnancy, which may be because those patients feared drug teratogenicity and had rarely consulted epileptologist before and during pregnancy for relevant knowledge of epilepsy and AED compliance. This absence AED use may also explain the greater number of GTCS attacks in the unplanned-pregnancy group.

The most commonly used AEDs as monotherapy in first trimester were LTG, LEV, and OXC in the planned group, which is in accordance with current guidelines and opinions. ${ }^{14,} 26,27$ However, in the unplanned group, 
LTG and VPA were among the medications most commonly prescribed. In Shaanxi Province, in the last decade, we have been implementing a program on rational drug selection for WWE. This program could make many WWE in this study be prescribed with LTG at the onset of illness. VPA is a broad-spectrum AED with potent antiepileptic effects in WWE. ${ }^{23,}{ }^{24}$ Additionally, it is the most teratogenic AED, with a several-fold increase in MCMs risk. ${ }^{15}, 16,25$ In the planned-pregnancy group, no WWE took VPA as monotherapy. This finding could also be attributed to the promotion of our program.

Compared with planned-pregnancy group, the unplanned group had a relatively high rate of induced abortions. This choice was likely made because of the following reasons: 1) fear of the effect of frequent seizures on the fetus; 2) fear of AEDs teratogenicity, or 3) abnormal prenatal examination. In addition, the unplanned group tended to have a greater rate of SFL, which could be also attributed to more GTCS attacks in the unplanned-pregnancy group.

Adverse fetal outcomes of WWE mainly include MCMs, cognitive outcomes such as impaired intellectual and verbal performance, autism spectrum disorder, and growth restriction (i.e., being small for gestational age or smaller than normal head circumference). ${ }^{23,}{ }^{28-30}$ Our study focused on MCMs. MCMs are defined as structural abnormalities with significant interference with function and/or require surgery for correction. ${ }^{31,} 32$ Results from Swedish registries demonstrated that among the 1,429,652 included births, MCM prevalence among AED-exposed offspring was $6.7 \%$ and was $4.7 \%$ in offspring of WWE without AED treatment. ${ }^{4}$ Recent data from the European and International Registry of Antiepileptic Drugs in Pregnancy (EURAP) showed that prevalence of MCMs in offspring exposed prenatally to AED monotherapy ranged from $2.8 \%$ to $10.3 \%$, depending on the type of AEDs. ${ }^{15}$ In the Maternal Outcomes and Neurodevelopmental Effects of Antiepileptic Drugs study, adverse fetal outcomes including fetal loss and MCMs were significantly more common in WWE than in healthy pregnant women. ${ }^{33}$

In our study, the MCM rate was significantly lower in the planned-pregnancy group (1.6\%) than in the unplanned-pregnancy group (7.5\%). This decrease in MCMs rate could be partly because of the relatively less use of VPA, PB, phenytoin (PHT), CBZ, and topiramate (TPM) in the planned-pregnancy group than in the unplanned group. The five aforementioned AEDs were reported in the EURAP registry to be related to a high prevalence of MCMs. ${ }^{15}$ For types of MCM, cardiac defect was most common in both the planned and unplanned group, which was consistent with the finding of the EURAP registry. ${ }^{15}$ However, in the unplanned-pregnancy group, except for cardiac defect, there were cases of bifid spine, cleft lip/palate, hypospadias, conjoined twins, and anotia. Encouragingly, the planned-pregnancy group only had an MCM prevalence of $1.6 \%$, which indicates that optimized pregnancy management can significantly decrease the risk of offspring adverse outcomes.

This study has several limitations. First, our study assessed a relatively small sample and was conducted at a single site in a university hospital setting. Thus, validation of our findings in different areas and with a large sample would be necessary to better define the importance of pregnancy planning. Second, instead of all types of seizures, we recorded only the number of GTCS during pregnancy, which has been reported to have significant negative effects on the offspring of WWE. Notably, because of the nature of this retrospective study, recording an accurate number of seizures other than GTCS was difficult. Third, detailed information on neonates such as birth weight and Apgar score was not available at the time of our study, which prevented comprehensive analysis of the effect of pregnancy planning on neonatal outcomes. Our study also did not record minor congenital malformations such as ocular hypertelorism, lop ear, hypoplastic fingernails, and ankyloglossia, etc. In addition, tobacco smoking and alcohol consumption during pregnancy were not recorded. These factors have also been reported to affect fetal outcomes. ${ }^{34,35}$

\section{Conclusions}

In conclusion, our study investigated the clinical characteristics and fetal outcomes of WWE in planned and unplanned pregnancy. We found that WWE with a planned pregnancy were associated with more optimized AED treatment patterns, better seizure control, more appropriate folic acid supplementation, and less fetal MCMs. Our study further verified the necessity and importance of pregnancy planning in the effective 
management of WWE of childbearing age. Further research with a larger sample size and comprehensive information on neonatal outcomes is necessary.

Acknowledgements: None.

Disclosure of Interests: None of the authors has any conflict of interest to disclose.

Contribution to authorship: YYZ had full access to all of the data in the study, conceived and designed the study, analysed and interpreted the data and drafted the manuscript. YYZ and CGS both contributed equally to the research study and manuscript. XW interpreted the data and revised the article. WJ conceived and designed the study, interpreted the data and revised the article. YLJ, JJZ, FY, XAY and FY interpreted the data and revised the article. All authors approved the final version.

Details of ethics approval: This study was approved by the Ethics Committee of Xijing Hospital (KY20163380 -1).

Funding: This study was supported by the National Natural Science Foundation of China (Grant Numbers 81771406 to Wen Jiang \& Grant Numbers 81974204 to Wen Jiang).

\section{References:}

1. World Health Organization. Epilepsy: Fact sheet. World Health Organization, 2017. Available athttp://www.who.int/mediacentre/factsheets/fs999/en/ . Accessed November 3, 2017.

2. Artama M, Braumann J, Raitanen J, Uotila J, Gissler M, Isojarvi J, et al. Women treated for epilepsy during pregnancy: outcomes from a nationwide population-based cohort study. Acta Obstet Gynecol Scand. 2017 Jul;96(7):812-20.

3. MacDonald SC, Bateman BT, McElrath TF, Hernandez-Diaz S. Mortality and Morbidity During Delivery Hospitalization Among Pregnant Women With Epilepsy in the United States. JAMA Neurol. 2015 Sep;72(9):981-8.

4. Razaz N, Tomson T, Wikstrom AK, Cnattingius S. Association Between Pregnancy and Perinatal Outcomes Among Women With Epilepsy. JAMA Neurol. 2017 Aug 1;74(8):983-91.

5. Stephen LJ, Harden C, Tomson T, Brodie MJ. Management of epilepsy in women. The Lancet Neurology. 2019;18(5):481-91.

6. Soontornpun A, Choovanichvong T, Tongsong T. Pregnancy outcomes among women with epilepsy: A retrospective cohort study. Epilepsy Behav. 2018 May;82:52-6.

7. Hernandez-Diaz S, Smith CR, Shen A, Mittendorf R, Hauser WA, Yerby M, et al. Comparative safety of antiepileptic drugs during pregnancy. Neurology. 2012 May 22;78(21):1692-9.

8. Tomson T, Battino D, Bonizzoni E, Craig J, Lindhout D, Sabers A, et al. Dose-dependent risk of malformations with antiepileptic drugs: an analysis of data from the EURAP epilepsy and pregnancy registry. Lancet Neurol. 2011 Jul;10(7):609-17.

9. Meador KJ, Baker GA, Browning N, Cohen MJ, Bromley RL, Clayton-Smith J, et al. Fetal antiepileptic drug exposure and cognitive outcomes at age 6 years (NEAD study): a prospective observational study. The Lancet Neurology. 2013;12(3):244-52.

10. Kilic D, Pedersen H, Kjaersgaard MI, Parner ET, Vestergaard M, Sorensen MJ, et al. Birth outcomes after prenatal exposure to antiepileptic drugs-a population-based study. Epilepsia. 2014 Nov;55(11):1714-21.

11. Bibbins-Domingo K, Grossman DC, Curry SJ, Davidson K, Epling JW, Garcia FAR, et al. Folic Acid Supplementation for the Prevention of Neural Tube Defects US Preventive Services Task Force Recommendation Statement. Jama-J Am Med Assoc. 2017 Jan 10;317(2):183-9. 
12. Bjork M, Riedel B, Spigset O, Veiby G, Kolstad E, Daltveit AK, et al. Association of Folic Acid Supplementation During Pregnancy With the Risk of Autistic Traits in Children Exposed to Antiepileptic Drugs In Utero. JAMA Neurol. 2018 Feb 1;75(2):160-8.

13. Sveberg L, Svalheim S, Tauboll E. The impact of seizures on pregnancy and delivery. Seizure. 2015 May;28:35-8.

14. Vajda FJ, O'Brien T, Lander C, Graham J, Eadie M. The efficacy of the newer antiepileptic drugs in controlling seizures in pregnancy. Epilepsia. 2014 Aug;55(8):1229-34.

15. Tomson T, Battino D, Bonizzoni E, Craig J, Lindhout D, Perucca E, et al. Comparative risk of major congenital malformations with eight different antiepileptic drugs: a prospective cohort study of the EURAP registry. Lancet Neurology. 2018 Jun;17(6):530-8.

16. Veroniki AA, Cogo E, Rios P, Straus SE, Finkelstein Y, Kealey R, et al. Comparative safety of antiepileptic drugs during pregnancy: a systematic review and network meta-analysis of congenital malformations and prenatal outcomes. BMC Med. 2017 May 5;15(1):95.

17. Pennell PB. Use of Antiepileptic Drugs During Pregnancy: Evolving Concepts. Neurotherapeutics. 2016 Oct;13(4):811-20.

18. Herzog AG, Mandle HB, Cahill KE, Fowler KM, Hauser WA. Predictors of unintended pregnancy in women with epilepsy. Neurology. 2017 Feb 21;88(8):728-33.

19. Herzog AG, Mandle HB, MacEachern DB. Association of Unintended Pregnancy With Spontaneous Fetal Loss in Women With Epilepsy Findings of the Epilepsy Birth Control Registry. Jama Neurology. 2019 Jan;76(1):50-5.

20. Scheffer IE, Berkovic S, Capovilla G, Connolly MB, French J, Guilhoto L, et al. ILAE classification of the epilepsies: Position paper of the ILAE Commission for Classification and Terminology. Epilepsia. 2017 Apr;58(4):512-21.

21. Obstetrics Subgroup CSoOaG CMA. Guideline of preconception and prenatal care(2018). . Zhonghua fu chan ke za zhi 2018;2018;53(1):7-13.

22. Johnson EL, Burke AE, Wang A, Pennell PB. Unintended pregnancy, prenatal care, newborn outcomes, and breastfeeding in women with epilepsy. Neurology. 2018 Sep 11;91(11):e1031-e9.

23. Tomson T, Battino D. Teratogenic effects of antiepileptic drugs. Lancet Neurology. 2012 Sep;11(9):80313.

24. Singh KP VN. Teratogenic potential of third-generation antiepileptic drugs: Current status and research needs. Pharmacological reports : PR. 2019;2019;71(3):491-502.

25. Keni RR, Jose M, Sarma PS, Thomas SV, Kerala Registry of E, Pregnancy Study G. Teratogenicity of antiepileptic dual therapy: Dose-dependent, drug-specific, or both? Neurology. 2018 Feb 27;90(9):e790-e6.

26. Patsalos PN BD, Bourgeois BF, et al. . Antiepileptic drugs-best practice guidelines for therapeutic drug monitoring: a position paper by the subcommission on therapeutic drug monitoring, ILAE Commission on Therapeutic Strategies. . Epilepsia 2008;2008;49(7):1239-1276.

27. Kim H, Faught E, Thurman DJ, Fishman J, Kalilani L. Antiepileptic Drug Treatment Patterns in Women of Childbearing Age With Epilepsy. JAMA Neurol. 2019 Apr 1.

28. Artama M GM, Malm H, Ritvanen A, Drug, Pregnancy G. . Effects of maternal epilepsy and antiepileptic drug use during pregnancy on perinatal health in offspring: nationwide, retrospective cohort study in Finland. Drug Saf. 2013;2013;36(5):359-369.

29. Veiby G DA, Engelsen BA, Gilhus NE. Fetal growth restriction and birth defects with newer and older antiepileptic drugs during pregnancy. . Journal of neurology 2014; 2014;261(3):579-588. 
30. Almgren M KB, Lavebratt C. . Population-based study of antiepileptic drug exposure in utero-influence on head circumference in newborns. Seizure. 2009;2009;18(10):672-675.

31. Holmes LB HE, Coull BA, et al. . The teratogenicity of anticonvulsant drugs. N Engl J Med. 2001; 2001;344(15):1132-1138.

32. Voinescu PE, Pennell PB. Management of epilepsy during pregnancy. Expert Rev Neurother. 2015 Oct;15(10):1171-87.

33. Meador KJ PP, May RC, et al. . Fetal loss and malformations in the MONEAD study of pregnant women with epilepsy. NEUROLOGY. 2019;2019.

34. Gustavson K YE, Stoltenberg C, et al. . Smoking in Pregnancy and Child ADHD. . Pediatrics $2017 ; 2017 ; 139(2)$.

35. Caputo C WE, Jabbour L. . Impact of fetal alcohol exposure on body systems: A systematic review. . Birth defects research Part C, Embryo today : reviews 2016; 2016;108(2):174-180.

\section{Hosted file}

Tables.docx available at https://authorea.com/users/303706/articles/433860-clinicalcharacteristics-and-fetal-outcomes-in-women-with-epilepsy-with-planned-and-unplannedpregnancy-a-retrospective-study

\section{Hosted file}

Figures.docx available at https://authorea.com/users/303706/articles/433860-clinicalcharacteristics-and-fetal-outcomes-in-women-with-epilepsy-with-planned-and-unplannedpregnancy-a-retrospective-study 